\title{
riccafd
}

Revista Iberoamericana de Ciencias de la Actividad Física y el Deporte

\section{ANÁLISIS DE LA COLOCACIÓN EN SETS \\ DE DIFERENTE INTENSIDAD, EN EL U-21 MEN'S WORLD CHAMPIONSIP DE VOLEIBOL}

\section{ANALYSIS OF THE SET IN SETS WITH VARIED FINAL SCORE DIFFERENCE, IN THE U-21 MEN'S WORLD CHAMPIONSIP VOLLEYBALL}

González-Silva, J. ${ }^{1}$ Fernández-Echeverría, C. ${ }^{2}$, Claver, F. ${ }^{3}$ y Moreno, M.P. ${ }^{4}$

${ }^{1}$ Estudiante de doctorado de la de la Universidad de Extremadura.

Facultad de Ciencias del Deporte de Cáceres (España).jarags@unex.es

${ }^{2}$ Personal Docente Investigador de Universidad de Sevilla.

Facultad de Ciencias de la Educación de Sevilla (España). cfecheverria@us.es

${ }^{3}$ Profesor Sustituto Interino de la Universidad de Extremadura.

Facultad de Ciencias del Deporte (España). fclaver@unex.es

${ }^{4}$ Profesora Titular de la Universidad de Extremadura.

Facultad de Ciencias del Deporte de Cáceres (España).pmoreno@unex.es

Correspondencia: González-Silva, J.jarags@unex.es

Código UNESCO: 5899 Otras Especialidades Pedagógicas (Educación Física y Deporte)

Clasificación Consejo de Europa: 16. Sociología del deporte

Recibido el 5 de octubre de 2018

Aceptado el 2 de abril de 2019

DOI: http://dx.doi.org/10.24310/riccafd.2019.v8i2.6451

Agradecimientos:

Este trabajo ha sido desarrollado gracias a financiación otorgada por la fundación Tatiana Pérez de Guzmán el Bueno y gracias a la aportación de la Consejería de Economía e Infraestructuras de la Junta de Extremadura a través del Fondo Europeo de Desarrollo Regional.

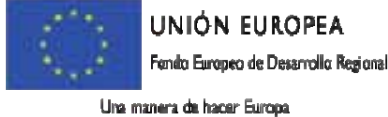

Une mantera de hacor Europa
JUNTA DE EXTREMADURA

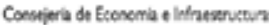

\section{RESUMEN}

El objetivo de la presente investigación fue conocer las variables de la colocación que afectan al desarrollo del rally, en sets de diferente intensidad, en voleibol masculino U-21. La muestra del estudio estuvo compuesta por 1688 acciones de colocación que se desarrollaron en el complejo 1 (KI) (1100 en sets 
de intensidad alta y 588 en sets de intensidad no alta), realizadas por las cuatro selecciones mejor clasificadas en el U-21 Men's World Championsip de voleibol. La observación correspondió a cinco partidos de cada una de las selecciones participantes. Las variables del estudio fueron: origen del colocador, zona de colocación, tipo de colocación, técnica de colocación, eficacia de colocación, zona hacia donde se envía la colocación, tiempo de colocación, desarrollo del rally, e intensidad del set. Los resultados de la regresión logística multinomial mostraron que, tanto en los sets de intensidad alta como en los de intensidad no alta, el tipo de colocación y la técnica de colocación fueron predictoras del desarrollo del rally. Además, en los sets de intensidad alta, la zona y eficacia de colocación también actuaron como predictoras del desarrollo del rally. En los sets de intensidad no alta, la zona hacia donde se envía la colocación actuó como predictora del desarrollo del rally, no siendo predictora en los sets de intensidad alta. Dichos resultados pueden ser considerados para el diseño de tareas específicas de entrenamiento de la colocación, acordes a diferentes situaciones de juego.

Palabras clave: variables situacionales, voleibol, colocación, masculino.

\section{ABSTRACT}

The aim of the study was to determine the setting variables that predict the rally development in men's U-21 volleyball, in sets with varied final score difference. The study sample comprised 1688 setting actions in Complex I (KI) (1100 in sets with high final score difference and 588 in sets with not high final score difference), made by the four top ranked teams in the Volleyball Men's U-21 World Championship. Five matches from each team were observed. The variables of the study were: setter position, setting zone, type of set, setting technique, setting efficacy, set's area, set tempo, development of rally, set final score difference. The multinomial logistic regression showed that, both in sets with high final score difference as in sets with not high final score difference, type of set and setting technique predicted the development of the rally. Moreover, in sets with high final score difference, setting zone and setting efficacy predicted the development of rally. In sets with not high difference of final score, set's area predicted the development of the rally. These results can be considered to design specific training tasks of the set, according to the different game situations.

Key words: situacional variables, volleyball, set, male.

\section{INTRODUCCIÓN}

Las investigaciones sobre análisis del juego han ido evolucionando a lo largo del tiempo. En la actualidad, estos estudios han pasado de analizar únicamente las diferentes acciones de juego que acontecen en el deporte, a tener en cuenta el contexto, incluyendo las denominadas variables situacionales ${ }^{1-3}$. Entre ellas encontramos la localización del partido, la calidad del oponente, el resultado del 
partido, entre otras ${ }^{1}$. Dichas variables han sido analizadas en diferentes deportes como el baloncesto ${ }^{4}$, balonmano ${ }^{5}$.

Nuestro estudio, específicamente, se centrará en el deporte del voleibol. En voleibol, para ganar un partido uno de los equipos tiene que conseguir ganar tres sets de cinco, los cuales se ganan llegando a 25 puntos, con diferencia mínima de dos puntos. Esto hace que el set sea un elemento importante de análisis, centrándose en el mismo diversas investigaciones. Así, Ramos ${ }^{6}$, estudiaron la variabilidad del rendimiento táctico en diferentes niveles competitivos en función del periodo y el tipo de set (sets finalistas y sets no finalistas). Marcelino ${ }^{7}$, quisieron conocer la probabilidad de ganar o perder el set en función de la localización del partido y del resultado del set (ganados, perdidos). Campos $^{8}$, investigaron sobre la ventaja de jugar en casa y la influencia de los indicadores de rendimiento en la puntación, en función del número de sets jugados (tres, cuatro o cinco). Sin embargo, cuando nos centramos en la diferencia del marcador final en el set, el número de estudios se reduce considerablemente. Drikos ${ }^{9}$, en un estudio realizado en alto nivel, pretendieron identificar los indicadores de rendimiento de voleibol que mejor discriminaban entre los equipos ganadores y perdedores teniendo en cuenta la intensidad del set, considerando, la intensidad del set, como la diferencia del marcador final del set de los equipos participantes.

Por otro lado, en voleibol, las acciones de juego pueden ser de dos tipos, acciones finalistas (saque, ataque y bloqueo) y acciones intermedias (recepción, colocación y defensa). Las acciones finalistas son aquellas a través de las cuales se obtiene punto ${ }^{10}$, siendo las intermedias aquellas a través de las cuales no se suele conseguir punto ${ }^{11}$. A pesar de ello, las acciones intermedias son importantes en el juego, al actuar de enlace entre las finalistas ${ }^{12}$, y por afectar, la calidad de estas acciones, a la eficacia de las acciones finalistas ${ }^{13}$. Por ello, las acciones intermedias pueden, incluso, influir en el rendimiento final ${ }^{14}$. De estas acciones, nuestro estudio se centrará en la colocación.

La colocación tiene como principal objetivo dejar al atacante en las mejores condiciones para realizar el ataque ${ }^{15}$. La colocación es realizada por un jugador especializado, el colocador, y es el segundo contacto que lleva a cabo el equipo. La colocación, es una de las acciones más importantes, por ser a través de la cual se organiza la ofensiva del equipo ${ }^{14}$, y por depender, un alto porcentaje de la eficacia del ataque, de la calidad de la colocación ${ }^{16}$. Pero, además de influir la colocación en el ataque, ésta se ve afectada por las acciones previas como el saque y la recepción ${ }^{17}$. Concretamente, la recepción, puede afectar tanto a la zona donde el colocador realiza la colocación, como a la eficacia de la colocación ${ }^{18}$. Uno de los aspectos necesarios para que la recepción influya menos sobre la eficacia de la colocación es que los colocadores tengan un elevado dominito técnico. Con ello, podrán realizar colocaciones buenas independientemente de las condiciones en las que les llega el balón ${ }^{17}$. Esto hace que la colocación sea una de las primeras acciones en las que se inicia la especialización ${ }^{19}$.

Por la importancia de la colocación y el reducido número de investigaciones realizadas en las que se tiene en cuenta variables situacionales como la intensidad del set, el objetivo de la presente investigación es conocer las variables de 
la colocación que afectan al desarrollo del rally, en sets de diferente intensidad, en voleibol masculino U-21.

\section{MÉTODO}

\section{Participantes}

La muestra del estudio estuvo compuesta por 1688 acciones de colocación en el KI, de las cuales 1100 se realizaron en sets de intensidad alta y 588 en sets de intensidad no alta, correspondientes a la observación de las cuatro selecciones mejor clasificadas en el U-21 men's World Championship. La observación corresponde a cinco partidos, de cada equipo, lo que supone la observación de 53 sets.

\section{Variables}

Las variables del estudio fueron las siguientes:

Origen del colocador: definido como la posición en la que se encuentra el jugador que realiza el segundo pase de colocación. Se utilizaron las categorías empleadas por Palao ${ }^{20}$ : colocador zaguero, cuando el colocador se encuentra en la zona 1, 6 ó 5; colocador delantero, cuando el colocador se encuentra en la zona 4, 3 ó 2.

Zona de colocación: definido como el lugar del campo desde donde se realiza el pase de colocación. Se utilizaron las categorías empleadas por Afonso ${ }^{21}$ : zona excelente/zona $\mathrm{A}$, área de $10 \mathrm{~m}^{2}, 5$ metros de largo por 2 metros de ancho, ubicada a 1 metro de la línea lateral derecha y a 3 metros de la línea lateral izquierda; zona aceptable/zona $B$, área de $6 \mathrm{~m} 2$, a 2 metros de la zona $A$ y 3 metros de ancho, ubicado a 2 metros de la línea lateral derecha y a 4 metros de la línea lateral izquierda; zona no aceptable/zona $\mathrm{C}$, que equivale a todo el área de juego excluidas las zonas A y B (Figura 1).

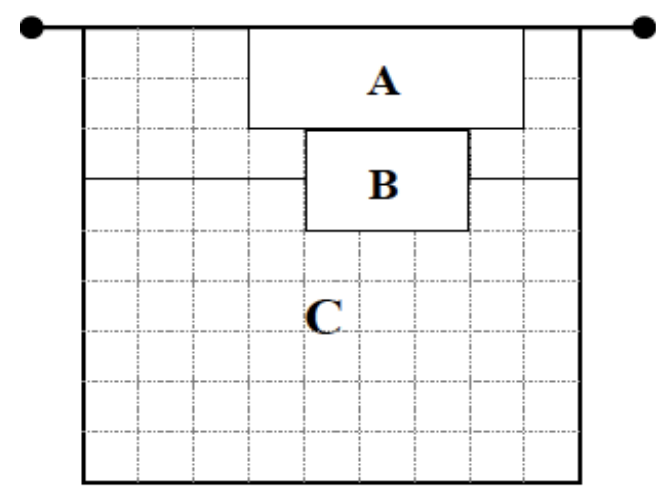

Figura 1. Zona de colocación ${ }^{21}$. 
Tipo de colocación: definido como la colocación que realiza el jugador, considerando si el colocador se encuentra en contacto con el suelo o no, en el momento de realizar la colocación. Se utilizaron las categorías empleadas por Palao ${ }^{15}$ : colocación en salto, cuando el colocador tiene los pies en el aire en el momento de contactar con el balón; colocación en apoyo, cuando el colocador tiene los pies tocando el suelo en el momento de contactar con el balón.

Técnica de colocación: definida como el gesto técnico empleado en el pase de colocación. Para ello se utilizaron las categorías establecidas por Papadimitriou ${ }^{17}$ : colocación de dedos, el colocador realiza la colocación con las dos manos; colocación de antebrazos, el colocador realiza la colocación con los antebrazos.

Eficacia de la colocación: definida como el rendimiento o efecto obtenido en la colocación. Se utilizaron las categorías del Sistema FIVB, adaptado de Coleman $^{22}$, agrupando algunos valores que aparecían diferenciados en dicho sistema. Las categorías fueron: colocación no precisa, definida como la colocación que no permite realizar un ataque, o la colocación que permite realizar un ataque, pero sin todas las opciones; colocación buena, definida como la colocación precisa que permite ataque ante dos o un bloqueador y medio; colocación perfecta, definida como la colocación precisa que permite el ataque ante uno o ningún bloqueador (adaptado de Moreno ${ }^{23}$ ).

Zona hacia donde se envía la colocación: definido como la zona o lugar del campo donde se realiza la batida para el ataque. Fueron utilizadas las categorías empleadas por Papadimitriou ${ }^{17}$ : zona zaguera, zona 4, zona 3, zona 2 (Figura 2).

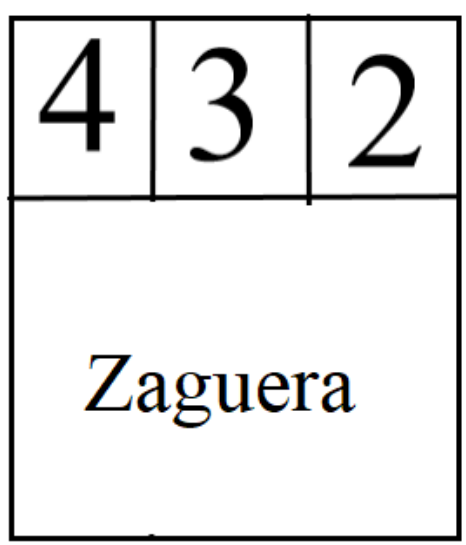

Figura 2. Zona hacia donde se envía la colocación

Tiempo de colocación: definido como la interacción entre el momento en el que el colocador contacta con el balón y el inicio de aproximación del atacante. Se utilizó una adaptación de las categorías empleadas por Afonso ${ }^{18}$ y Papadimitriou ${ }^{17}$ : primer tiempo, el atacante está en el aire cuando el balón llega al colocador; segundo tiempo, el atacante está realizando el penúltimo paso de la carrera cuando el balón llega al colocador; tercer tiempo, el atacante no ha iniciado la carrera de ataque cuando el balón llega al colocador. 
Desarrollo del rally, definido como el desenvolvimiento del rally, considerando si finaliza o no en $\mathrm{KI}$, o si el rally continúa. Las categorías fueron: error, el rally termina en el complejo $\mathrm{KI}$, perdiendo el punto el equipo que realizó la colocación; continuidad, el rally continúa no finalizando en el complejo KI; punto, el rally finaliza en el complejo KI, ganando el punto el equipo que realizó la colocación.

Intensidad del set, definida como la diferencia en la puntuación final del set. Se utilizó una adaptación de las categorías empleadas por Sánchez-Moreno ${ }^{24}$ : intensidad alta, una diferencia menor o igual de cinco puntos en el resultado final del set; intensidad no alta, una diferencia mayor de cinco puntos en el resultado final del set.

\section{Procedimiento}

Las grabaciones de los partidos fueron realizadas con una cámara digital SONY HDR-XR155 sobre formato M2TS. Dicha cámara se ubicaba en uno de los fondos de la cancha de juego para obtener un óptimo plano de visión.

Una vez realizada la recogida de datos se procedió a la observación de todos los partidos por un único observador. Para garantizar la fiabilidad de la observación, un observador con las siguientes características, Licenciado en Ciencias de la Actividad Física y el Deporte, entrenador Nacional de Nivel III de voleibol, y con cinco años de experiencia como entrenador, realizó un proceso de entrenamiento con muestras con características diferentes (intensidad del set, clasificación de los equipos en competición, set en juego). Se alcanzaron en la observación de todas las variables unos valores de Kappa de Cohen intra-observador superiores a .75 , valor mínimo a partir del cual se considera una concordancia casi perfecta ${ }^{25}$. Para garantizar la fiabilidad temporal de la medida, se desarrolló la misma observación en dos ocasiones, con una diferencia temporal de diez días, obteniendo unos valores de Kappa de Cohen superiores a .75.

\section{Análisis Estadístico}

Se realizó un análisis inferencial para comprobar las asociaciones entre cada una de las variables estudiadas y el desarrollo del rally. Este análisis se presenta a través de las tablas de contingencia, incluyendo los valores de Chi-Cuadrado y $V$ de Cramer, con corrección de Monte Carlo. El nivel de significación estadística que se consideró fue de $p<.05$. Posteriormente, a través del modelo de regresión logística multinomial, se obtuvieron las predicciones del desarrollo del rally, respecto a cada una de las variables del estudio. Previo al análisis de regresión, para evitar la inclusión de variables intercorrelacionadas, realizamos una prueba de multicolinealidad. Este análisis mostró que no existía multicolinealidad entre las variables al no obtener en ninguna de ellas valores de Tolerancia $>0.10 \mathrm{y}$ valores de VIF $<10^{26}$. Por tanto, todas las variables fueron incluidas en el modelo de regresión. 


\section{RESULTADOS}

\section{Análisis inferencial}

A través del análisis inferencial se muestran las asociaciones obtenidas entre cada una de las variables de estudio y el desarrollo del rally, en las dos intensidades del set (alta y no alta), indicando los valores de Chi-cuadrado y $\mathrm{V}$ de Cramer

Los resultados mostraron tanto en los sets de intensidad alta como en los de intensidad no alta, una asociación significativa entre el desarrollo del rally y la zona de colocación, tipo de colocación, técnica de colocación, eficacia de colocación, zona hacia donde se envía la colocación y tiempo de colocación. Por el contrario, únicamente no existió asociación significativa, en ambas intensidades, entre el desarrollo del rally y el origen del colocador. Por tanto, dicha variable no pudo ser incluida en el modelo de regresión logística multinomial (Tabla 1).

Tabla 1. Asociación del desarrollo del rally con las variables del estudio, en las dos intensidades del set (alta y no alta).

\begin{tabular}{|c|c|c|c|c|c|c|}
\hline \multirow[b]{2}{*}{ Variables } & \multicolumn{3}{|c|}{ Intensidad alta } & \multicolumn{3}{|c|}{ Intensidad no alta } \\
\hline & p. & $x^{2}$ & $\begin{array}{l}\mathrm{V} \\
\text { Crame }\end{array}$ & dep. & $X^{2}$ & V de Cramer \\
\hline Origen del colocador & .941 & .122 & .011 & .301 & 2.399 & .064 \\
\hline Zona de colocación & .000 & 47.829 & .147 & .000 & 20.141 & .131 \\
\hline Tipo de colocación & .000 & 33.961 & .176 & .000 & 32.956 & .237 \\
\hline Técnica de colocación & .000 & 37.182 & .184 & .000 & 30.631 & .228 \\
\hline Eficacia de colocación & .000 & 58.917 & .164 & .000 & 22.590 & .139 \\
\hline $\begin{array}{l}\text { Zona hacia donde } \\
\text { se envía la colocación }\end{array}$ & .005 & 18,577 & .092 & .024 & 14.572 & .111 \\
\hline Tiempo de colocación & .001 & 19.050 & .093 & .024 & 11,276 & .098 \\
\hline
\end{tabular}

\section{Análisis predictivo}

En los sets de intensidad alta (Tabla 2), en la comparación entre el punto y la continuidad en el rally, el tipo de colocación, la técnica de colocación y la eficacia de colocación mostraron ser predictores del desarrollo del rally. Concretamente, en dichos sets, la colocación en salto, en lugar de en apoyo, redujo la frecuencia $(\mathrm{OR}=.603)$ de que el rally continúe, en lugar de que fuese punto. Además, las colocaciones de antebrazos, en lugar de las de dedos, incrementaron la frecuencia $(\mathrm{OR}=1.734)$ de que el rally continúe, en lugar de que terminase en punto. Finalmente, en relación a la eficacia de colocación, la eficacia de colocación no precisa y buena, en lugar de perfecta, incrementaron la frecuencia $(O R=1.912$ y 
$\mathrm{OR}=1.587$, respectivamente) de que el rally continúe, en lugar de que terminase en punto.

En la comparación entre el punto y el error en el rally, en los sets de intensidad alta, los resultados mostraron que, únicamente, la zona de colocación mostró ser predictora del desarrollo del rally. Ante colocaciones desde zonas aceptables, en lugar de zonas excelentes, era más frecuente $(\mathrm{OR}=2.761)$ que el rally finalizase en error, en lugar de punto (Tabla 2).

Tabla 2. Modelo de regresión del desarrollo del rally, en los sets de intensidad alta.

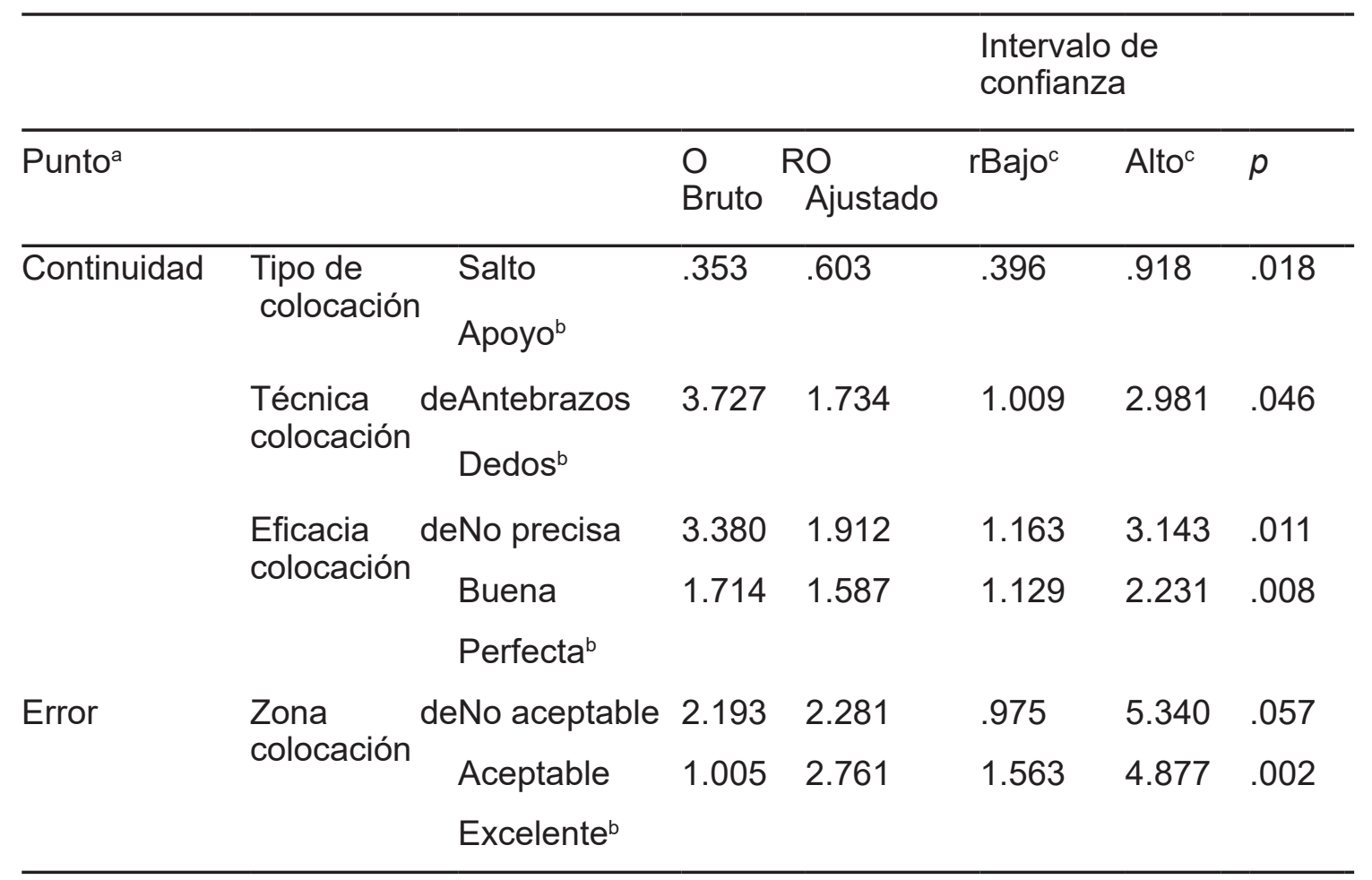

"a"Categoría de referencia de la variable dependiente. "b" Categoría de referencia de las variables independientes. "c"Intervalos de confianza 95\%.

En los sets de intensidad no alta (Tabla 3), en la comparación entre el punto y la continuidad en el rally, el tipo de colocación, técnica de colocación y zona hacia donde se envía la colocación mostraron ser predictores del desarrollo del rally. Concretamente, las colocaciones en salto, en lugar de en apoyo, descendieron la frecuencia $(\mathrm{OR}=.428)$ de que el rally continúe, en lugar de que terminase en punto. Además de ello, las colocaciones de antebrazos, en lugar de dedos, aumentaron la frecuencia $(O R=2.441)$ de que el rally continúe, en lugar de que finalizase con punto. Finalmente, el envío de colocaciones hacia zona zaguera, zona dos y zona tres, en lugar de zona cuatro, aumentó la frecuencia de que el rally continúe $(\mathrm{OR}=2.356, \mathrm{OR}=2.154$ y $\mathrm{OR}=3.035$, respectivamente), en lugar de que terminase en punto.

Finalmente, en los sets de intensidad no alta, en la comparación entre el punto y el error en el rally, ninguna variable actuó como predictora. 
Tabla 3. Modelo de regresión del desarrollo del rally, en los sets de intensidad no alta.

\begin{tabular}{|c|c|c|c|c|c|c|c|}
\hline \multirow{2}{*}{\multicolumn{3}{|c|}{ Punto $^{a}$}} & \multicolumn{5}{|c|}{$\begin{array}{l}\text { Intervalo de } \\
\text { confianza }\end{array}$} \\
\hline & & & & RO & $r \overline{\text { Bajo }^{c}}$ & Alto ${ }^{c}$ & $p$ \\
\hline & & & Bruto & Ajustado & & & \\
\hline \multirow[t]{8}{*}{ Continuidad } & \multirow{2}{*}{$\begin{array}{l}\text { Tipo de } \\
\text { colocación }\end{array}$} & Salto & .242 & .428 & .230 & .798 & .008 \\
\hline & & Apoyo $^{b}$ & & & & & \\
\hline & \multirow{2}{*}{$\begin{array}{l}\text { Técnica de } \\
\text { colocación }\end{array}$} & Antebrazos & 4.895 & 2.441 & 1.111 & 5.365 & .026 \\
\hline & & Dedos $^{b}$ & & & & & \\
\hline & \multirow{4}{*}{$\begin{array}{l}\text { Zona hacia } \\
\text { donde se } \\
\text { envía la } \\
\text { colocación }\end{array}$} & Zaguera & 1.975 & 2.356 & 1.355 & 4.097 & .002 \\
\hline & & Dos & 1.868 & 2.154 & 1.259 & 3.683 & .005 \\
\hline & & Tres & 1.540 & 3.035 & 1.223 & 7.531 & .017 \\
\hline & & Cuatro $^{b}$ & & & & & \\
\hline
\end{tabular}

"a"Categoría de referencia de la variable dependiente. "b" Categoría de referencia de las variables independientes. "c"Intervalos de confianza 95\%.

\section{DISCUSIÓN}

El objetivo de la presente investigación fue conocer las variables de la colocación que afectan al desarrollo del rally, en sets de diferente intensidad, en voleibol masculino U-21.

Los resultados mostraron que, tanto en los sets de intensidad alta como en los de intensidad no alta, el tipo de colocación y la técnica de colocación fueron variables predictoras del desarrollo del rally.

En relación al tipo de colocación, tanto en los sets de intensidad alta como en los de intensidad no alta, la colocación en salto, en lugar de en apoyo, incrementó la frecuencia de que el rally terminase en punto, en lugar de que el rally continuase. En cuanto a la técnica de colocación, en ambas intensidades de sets (alta y no alta), los resultados mostraron que las colocaciones de antebrazos, en lugar de dedos, produjeron un aumento de los rallies que continuaban, en lugar de los que terminaban en punto. A pesar de que no hemos encontrado investigaciones previas que analicen cómo afecta al desarrollo del rally el tipo y técnica de colocación, en diferentes intensidades de set, en línea con nuestros resultados, investigaciones previas constataron que ante colocaciones en salto ${ }^{27}$ y de dedos ${ }^{28}$ se conseguían unos valores mayores de rendimiento en la propia acción, lo que podría favorecer que el rally terminase en punto.

A través de la colocación en salto, el colocador es capaz de reducir sus preíndices $^{29}$, dificultando la anticipación del bloqueo rival ${ }^{18}$, así como acelerar la velocidad del juego ${ }^{30}$. Todo eso posibilita que el colocador pueda dejar al atacante ante un menor número de bloqueadores, facilitando ello la consecución del 
punto, al ser el bloqueo individual menos eficaz que el que está formado por un mayor número de jugadores ${ }^{31}$. Unido a ello, Ramos ${ }^{32}$, en un estudio en el que analizaron la colocación en equipos masculinos de la Superliga brasileña, constataron que la técnica de dedos, en cuanto a la ejecución de la colocación, era la más precisa. Este aspecto puede ser el motivo por el cual, tanto en los sets de intensidad alta como en los de intensidad no alta, la tecnica de dedos favorezca que el rally termine en punto, en lugar de que el rally continúe.

En relación a los sets de intensidad alta, la zona de colocación y la eficacia de colocación fueron también variables predictoras del desarrollo del rally, no siendo predictoras, dichas variables en los sets de intensidad no alta.

En cuanto a la zona de colocación, las colocaciones realizadas desde zona aceptable, en lugar de excelente, producían un aumento del error en el rally, en lugar de que éste terminase en punto. González-Silva ${ }^{33}$, analizaron las variables que predecían la eficacia de la colocación en género masculino y femenino U-16. Dicho estudio mostró que la zona de colocación era una variable predictora de la eficacia de la colocación en el género masculino. En la zona excelente de colocación, el colocador no tiene ninguna limitación para realizar la colocación, teniendo limitaciones cuando el colocador sale de la misma ${ }^{17}$. Esto mismo ocurre cuando hablamos de eficacia de colocación, descendiendo la eficacia a medida que el colocador se va alejando de la zona ideal, por la relación de dependencia entre la eficacia y la zona de colocación ${ }^{34}$. Esto hace que, cuando el colocador realiza la colocación en zona aceptable, en lugar de excelente, pueda aumentar el número de errores en colocación, aspectos que pueden afectar al desarrollo del rally, como se pone de manifiesto en nuestros resultados.

En relación a la eficacia de colocación, cuando se realizaban colocaciones con eficacia no precisa y buena, en lugar de perfecta, se incrementaban el número de rallies que continuaban, en lugar de los que terminaban en punto, en los sets de intensidad alta. Resultados similares se obtuvieron en el estudio de González-Silva ${ }^{35}$, en categoría U-16, donde las colocaciones con eficacia baja se asociaron con la pérdida del set.

Drikos $^{9}$ obtuvieron que, en los sets de intensidad alta, el número de errores era menor, por arriesgar menos los equipos en sus acciones. Unido a ello, en los sets de intensidad alta, todos los puntos y acciones pueden ser muy decisivas ${ }^{36}$. Esto puede hacer que características concretas de la colocación afecten al desarrollo del rally en sets de intensidad alta, no repercutiendo en los de intensidad no alta donde la diferencia de nivel entre los equipos puede hacer que la colocación no sea tan determinante en el desarrollo del juego.

Finalmente, en los sets de intensidad no alta, únicamente la zona hacia donde se envía la colocación fue variable predictora del desarrollo del rally, no siendo predictora en los sets de intensidad alta. Concretamente, las colocaciones hacia zona zaguera, zona dos y zona tres, en lugar de hacia zona cuatro, producían un incremento de que el rally continuase, en lugar de que terminase en punto.

Las colocaciones hacia zona tres suelen realizarse cuando las condiciones son ideales, al necesitar una gran sincronización entre el colocador y el atacante 
central ${ }^{18}$. Además de ello, en la zona cuatro, suele situarse el atacante más completo y regular del equipo. Por lo que, todo ello puede hacer que el envío hacia la zona cuatro, tal como muestra Costa $^{37}$, sea la ejecución más dominada y la que los colocadores realizan con una mayor seguridad, pudiendo afectar ella al desarrollo del rally.

En los sets de intensidad no alta la diferencia en la puntuación final de los equipos es elevada (menor de 5 puntos). Esto supone cierta tranquilidad para el equipo que está por encima en el marcador, que puede arriesgar más ${ }^{9}$, o incluso poner a prueba actuaciones diferentes.

Concluyendo, en voleibol masculino de alto nivel de categoría U-21, tanto en sets de intensidad alta como no alta, dos variables fundamentales de la colocación, el tipo de colocación (salto) y la técnica de colocación (dedos), predicen positivamente el desarrollo del rally, incrementando los rallies que finalizan en $\mathrm{KI}$, ganando el punto el equipo que realizó la colocación. Por otro lado, únicamente en los sets de intensidad alta, donde la diferencia de puntuación entre los equipos es reducida, distintas variables de colocación, concretamente la zona de colocación (zona aceptable) y la eficacia de colocación (no precisa y buena) descienden los rallies que finalizan en punto en KI. Finalmente, únicamente en los sets de intensidad no alta, la zona hacia donde se envía la colocación (zona cuatro), es predictora del desarrollo del rally, incrementando los rallies que finalizan en punto en $\mathrm{KI}$, y descendiendo los que suponen continuidad del rally.

\section{CONCLUSIONES}

Los resultados obtenidos pueden orientar el proceso de entrenamiento de la colocación, considerando durante el mismo el empleo de tareas de entrenamiento que reproduzcan situaciones de juego que acontecen en competición (con diferentes puntuaciones de los equipos), en las que se incida en objetivos relacionados con las variables de colocación que se muestran como predictoras del desarrollo del rally en cada situación.

\section{BIBLIOGRÁFIA}

1. Fernández-Echeverria C, Mesquita I, González-Silva J, Claver F, Moreno MP. Match analysis within the coaching process: a critical tool to improve coach efficacy. International Journal of Performance Analysis in Sport. 2017; 17(1-2):149-163. http://dx.doi. org/10.1080/24748668.2017.1304073.

2. Gonçalves BV, Figueira BE, Macas V, Sampaio J. Effect of player position on movement behaviour, physical and physiological performances during an-11-a-side football game. Journal of Sports Sciences. 2014; 32(2):191-199. http://doi.org/10.1080/0 2640414.2013.816761.

3. Marcelino R, Sampaio J, Mesquita I. Attack and serve performances according to the match period an quality of opposition in elite volleyball matches. Journal of Strength 
and Conditioning Research. 2012; 26(12):3385-3391. http://doi.org/10.1519/ JSC.0b013e3182474269

4. Gómez MA, Pérez J, Molik B, Szyman RJ, Sampaio J. Performance analysis of elite men's and women's wheelchair basketball teams. Journal of Sports Sciences. 2014; 32(11):1066-1075.

5. Meletakos P, Bayios I. General trends in European Men's Handball: A longitudinal study. International Journal of Performance Analysis in Sport. 2010; 10(3):221-228.

6. Ramos A, Coutinho P, Silva P, Davids K, Mesquita I. How players exploit variability and regularity of game actions in female volleyball teams, European Journal of Sport Science. 2017; 1-19. http://dx.doi.org/10.1080/17461391.2016.1271459.

7. Marcelino R, Mesquita I, Sampaio J, Anguera MT. Ventajas de jugar en cada en voleibol de alto rendimiento. Revista de Psicología del Deporte. 2009; 18(2):181-196.

8. Campos F, Stranganélli L, Campos L, Pasquarelli B, Gómez MA. Performance indicators analysis at brazilian and italian womwn's volleyball leagues according to game location, game outcome, and set number. Perceptual \& Motor Skills. 2014; 118(2): 1-15.

9. Driko S, Vagenas G. Multivariate assessment of selected performance indicators in relation to the type and resulto $f$ a typical set in Men's Elite Volleyball. International Journal of Performance Analysis in Sport. 2011; 11: 85-95.

10. Mesquita I, Manso F, Palao J. Defensive participation and efficacy of the líbero in volleyball. Journal of Human Movement Studies. 2007; 52(2):95-108.

11. Conejero M, Claver F, Fernández-Echeverría C, González-Silva J, Moreno MP. Diseño y validación de un instrumento de observación para valorar la toma de decisiones en la acción de recepción en voleibol. Cultura, Ciencia y Deporte. 2017; 12:67-75.

12. Hale T. Do human movement scientists obey the basic tenets of scientific 300 inquiry? Quest. 2001; 53(2):202-215.

13. Gil A, Moreno MP, Moreno A, García-González L, Del Villar F. Estudio del saque en jóvenes jugadores/as de voleibol, considerando la eficacia y función en juego. Retos. 2011; 19:19-24.

14. Silva MA, Lacerda D, Joao PV. Match analysis of discrimination skills according to the setter attack zone position in high level volleyball. International Journal of Performance Analysis in Sport 2013; 13(2):452-460.

15. Palao JM, Martínez S. Utilización de la colocación en salto en función del nivel de competición en voleibol masculino. Revista Euroamericana de ciencias del deporte. 2013; 2(1): 43-49.

16. Bergeles N, Barzouka K, Elissavet N. Performance of male and female setters and attackers on Olympiclevel volleyball teams. International Journal of Performance Analysis in Sport. 2009; 9(1):141-148.

17. Papadimitriou K, Pashali E, Sermaki I, Mellas S, Papas M. The effect of the opponents' serve on the offensive actions of Greek setters in volleyball games. International Journal of Performance Analysis in Sport. 2004; 4(1):23-33.

18. Afonso J, Mesquita I, Marcelino R, da Silva JA. Analysis of the setter's tactical action in high-performance women a volleyball. Kinesiology. 2010; 42(1): 82-89.

19. Palao JM, Echeverría, C. Evolución de la utilización de la colocación en salto en el 
voleibol femenino y su efecto sobre el juego. Kronos, Rendimiento en el deporte. 2008; 7(13):35-44.

20. Palao JM, Arhabi-Faid I. Side-out success in relation to setter's position on court in women's college volleyball. International Journal of Applied Sports Sciences. 2011; 23(1):155-167.

21. Afonso J, Esteves F, Araujo R, Thomas L, Mesquita I. Tactical determinants of setting zone in elite men's volleyball. Journal of Sports Science and Medicine. 2011; 11(1): 64-70.

22. Coleman JE. A statistical evaluation of selected volleyball techniques at the 1974 World`s Volleyball Championships [Thesis Physical Education]. Brigham Young University; 1975.

23. Moreno MP, García de Alcaraz A, Moreno A, Molina JJ. Santos JA. Estudio de la dirección del saque en la superliga masculina de voleibol. Motricidad. European Journal of Human Movement. 2007; 18:111-134.

24. Sánchez-Moreno J, Afonso J, Mesquita I, Ureña A. Dynamics between playing activities and rest time in high-level men's volleyball. International Journal of Performancen Analysis in Sport. 2016; 16:317-331.

25. 25Fleiss J, Levin B, Paik M. Statistical methods for rates and proportions. NY: John Wiley \& Sons; 2003.

26. Hair JF, Black WC, Babin BJ, Anderson R.E. Multivariate Data Analysis (7th ed.). Harlow: Pearson; 2014.

27. González-Silva J, Moreno A, Fernández-Echeverría C, Claver F, Moreno MP. Análisis del tipo de colocación empleado en voleibol, en categoría cadete. Kronos. 2015;14(1).

28. Palao J, Manzanares P, Ortega E. Techniques used and efficacy of volleyball skills in relation to gender. International Journal of Performance Analysis in Sport. 2009; 9(2):281-293.

29. Hernandez E, Urena A, Miranda MT, Ona A. Kinematic analysis of volleyball setting cues that affect anticipation in blocking. Journal of Human Movement Studies. 2004; 47(4):285-302.

30. Buscà B, Febrer J. Temporal fight between the middle blocker and the setter in high level volleyball. Revista Internacional de Medicina y Ciencias de la Actividad Física y el Deporte. 2012; 12(46): 313-327.

31. Afonso J, Mesquita I, Palao JM. The relationship between spike tempo and zone on the number of blockers in a variety of men's national team game phases. International Journal of Volleyball Research, 2005;8(1):19-23.

32. Ramos MHKP, Nascimento JV, Donegá AL, Novaes AJ, Souza RR, Silva TJ, Lopes AS. Estructura interna das açoes d levantamento das equipes finalistas da superliga masculina de voleibol. Revista Brasileira de Ciencia e Movimento. 2004; 12(4):33-37.

33. González-Silva J, Moreno A, Fernández-Echeverría C, Claver F, Moreno MP. Variables predictoras de la colocación en el complejo de defensa en voleibol. Revista Internacional de Medicina y Ciencias de la Actividad Física y el Deporte. 2018.

34. Afonso J, Mesquita I. Determinants of block cohesiveness and attack efficacy in high-level women's volleyball. European Journal of Sport Science. 2011;11(1): 6975, https://doi.org/10.1080/17461391.2010.487114. 
35. González-Silva J, Moreno A, Fernández-Echeverría C, Claver F, Moreno MP. Características de la colocación en voleibol, en etapas de formación, en sets ganados y perdidos, Retos. 2016; 30:43-47.

36. Valhondo A, Fernández-Echeverría C, González-Silva J, Claver F, Moreno MP. Variables that predict serve efficacy in elite men's volleyball with diferrent qualityz of oposition sets. Journal of Human Kinetic. 2018; 61:167-177. https://doi.org/10.1515/ hukin-2017-0119.

37. Costa GC, Mesquita I, Greco PJ, Ferreria NN, Moraes JC. Relación entre el tipo, tiempo y el efecto del ataque en el voleibol femenino juvenil de alto nivel de competición. Motricidad. European Journal of Human Movement. 2010; 24:121-132. 